\title{
Smart-GFEM for Welding Simulation
}

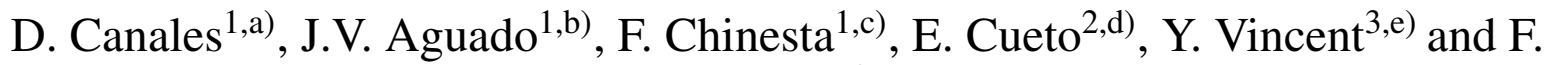 \\ Boitout $^{3, f)}$
}

${ }^{1}$ GeM, École Centrale de Nantes. 1 rue de la Noë, F-44321 Nantes, France.

${ }^{2}$ I3A, Universidad de Zaragoza. María de Luna 3, E-50018 Zaragoza, Spain.

${ }^{3}$ ESI Group, Le Récamier, 70 rue Robert, 69006 Lyon Cedex 06, France.

${ }^{a}$ Corresponding author: Diego.Canales-Aguilera@ec-nantes.fr

b) Jose.Aguado-Lopez@ec-nantes.fr

${ }^{c)}$ Francisco.Chinesta@ec-nantes.fr

d)ecueto@unizar.es

e)Yannick.Vincent@esi-group.com

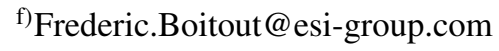

\begin{abstract}
In this paper, a new and efficient method for welding simulation is proposed. This method, known as Smart-GFEM introduces in a GFEM framework an enrichment function which is capable of adapting the approximation space optimally. The enrichment function can be seen as a computational vademecum which depends on technological and material parameters of the process and it is completely computed off-line. In this work we present how to perform the thermal analysis of the welding and a general strategy to address the mechanical analysis.
\end{abstract}

\section{INTRODUCTION}

Welding is a very complex process which involves a large number of physical phenomena that must be suitably modelled [1]. The most advanced simulation codes available are multiphysics, that is, they couple electromagnetic, thermal, metallurgical and mechanical models in order to obtain accurate results. Even if one assumes that these models are good enough to represent the physical process, the computational efficiency of the simulations must be improved to meet the requirements of modern industries. On one hand, traditional approaches are not competitive enough to explore the solution space if an important number of parameters are involved. On the other hand, new paradigms arise, such as data-driven simulations and augmented reality, where the numerical simulations should be performed in light computer platforms and deployed devices [2].

Model Order Reduction has formulated responses to these challenges, providing new powerful methods and strategies $[3,4]$ and also enriching traditional approaches to significantly improve their computational efficiency, as we do in this paper. We propose the Smart-GFEM [5] as an efficient global-local GFEM method [6] suitable for simulating processes with localized physics such as welding and heat treatments. The main strengths of Smart-GFEM are:

- The off-line computation of the local enrichment function.

- The conforming nature of the local problem.

- The explicit dependence of the enrichment function with respect to the technological and material parameters.

whilst preserving all the advantages of GFEM such us mesh-independent adaptivity [7] and allowance of a lowintrusive implementation in existing FEM codes [8]. 


\section{Smart-GFEM FOR THERMAL ANALYSIS}

The Generalized Finite Element Method (GFEM) was proposed by Melenk and Babuška [9]. The main idea of this method is to introduce the available information we have about the solution into the trial space. The GFEM is based in two properties: local approximability and conformity. This means that this method is able to construct a tailored approximation space with inter-element continuity.

For a given PDE, the GFEM approximation $u^{h}$ of the solution $u$ reads,

$$
u^{h}=\sum_{i \in I} N_{i}(\boldsymbol{x}) U_{i}+\sum_{e \in I_{e n r} \subset I} N_{e}(\boldsymbol{x}) \sum_{j} \phi^{j} b_{e}^{j},
$$

where the first term is a traditional FEM approximation, which satisfies the partition of unity $\sum_{i} N_{i}(x)=1, \forall x \in \Omega$, and the second one is the enrichment added. The set of nodes represented in $I$ and $I_{e n r}$ is the subset of enriched nodes. The functions $\phi^{j}$ are responsible for introducing the prior information we have of the solution, and therefore depend on the problem being solved.

The main issue of GFEM is selecting the set of enrichment functions. In the literature, analytical [10] and computational constructions can be found [11,6]. However, analytical constructions are not always available in practice and computational ones can be expensive for industrial applicability.

The Smart-GFEM proposes an original way of constructing the enrichment function, which is tailored to the problem in hand, being optimal at each instant of the simulation and having a negligible computational cost. Moreover, only one enrichment function is constructed, minimizing the computational cost of adding an enriched trial space.

In the Smart-GFEM, the enrichment function comes from a computational vademecum à la PGD [3] which contains the fundamental solution, $\phi$, of the localized physics of the problem for any values of the technological parameters, $p_{i}$, and for any essential boundary conditions, $u_{\Gamma}^{l}$, in its local domain. Thus, the Smart-GFEM approximation reads,

$$
u^{h}=\sum_{i \in I} N_{i}(\boldsymbol{x}) U_{i}+\sum_{e \in I_{e n r} \subset I} N_{e}(\boldsymbol{x}) \phi\left(\boldsymbol{x}, p_{1}, \ldots, p_{n}, u_{\Gamma}^{l}\right) b_{e} .
$$

For the sake of clarity, let us assume a thermal transient problem with a moving heat source $s(\boldsymbol{x}, t)$ in a domain $\Omega$ and in the temporal interval $\mathcal{T} \in(0, T)$,

$$
u_{t}-\nabla \cdot(k \nabla u)=s(\boldsymbol{x}, t) \quad \text { in } \quad \Omega \times \mathcal{T}
$$

where the main variable $u$ is the temperature and $k$ the thermal diffusivity. The essential and natural boundary conditions $u_{D}$ and $u_{N}$ are imposed on $\Gamma_{D}$ and $\Gamma_{N}$ respectively and the initial temperature is $u_{0}$.

Attached to the source, an enrichment region is set. The geometry of this region is fixed and, at each time step, the nodes located inside are affected with the optimal enrichment function that simply consists in particularizing the computational vademecum precomputed off-line as can be seen in Fig.1 The next parameters were introduced in the vademecum as extra-coordinates: the power of the heat source $Q$, the magnitude of the thermal source velocity $V$, the region of incidence of the heat source described by $\sigma$ and the temperature at the boundary of the enrichment region $u_{\Gamma e}$.

The vademecum is computed off-line and it is particularized on-line during the global simulation to obtain the optimal trial space in any instant. In Fig. 1, we can see that the enrichment function automatically adapts to the presence of a Dirichlet boundary condition $\Gamma_{D}$ along the path of the heat source in the enrichment region (dashed line).

Different tests were carried out, varying the technological parameters during the simulation. Very accurate results with the Smart-GFEM were obtained, even when a very coarse mesh was used. Fig.2 shows a comparison between the Smart-GFEM and a FEM solution, both computed in the same coarse mesh. If the area of incidence of the source significantly changes during simulation, the updating of the enrichment function is critical. Errors up to $25 \%$ in the maximum temperature in the thermal history of a material point were obtained when the enrichment function is not appropriately adapted. We can observed this effect in Fig.3, where the Smart-GFEM solution is updated to the parameter changes and the GFEM solution uses the same enrichment function during the complete simulation. 


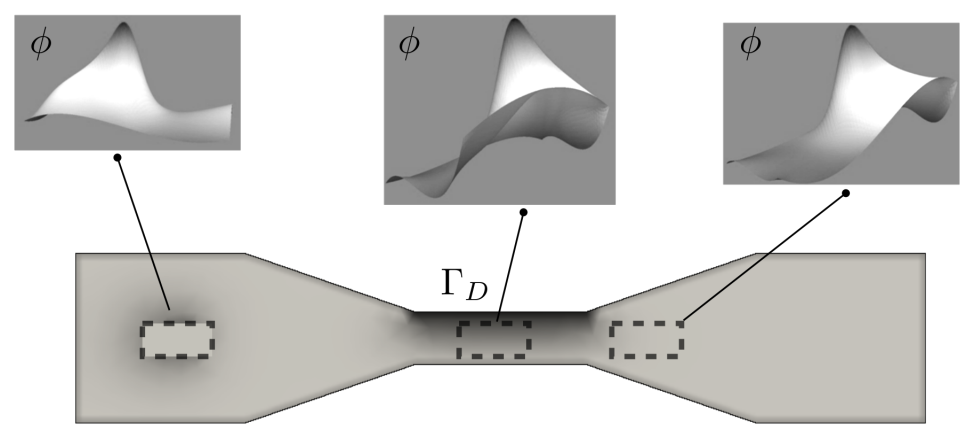

FIGURE 1: Updating the enrichment function

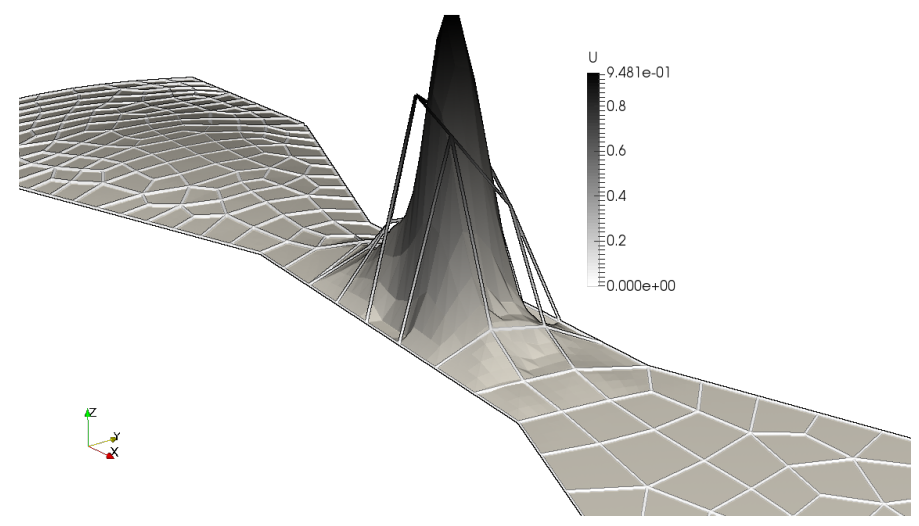

FIGURE 2: Smart-GFEM vs FEM in a coarse mesh

\section{Smart-GFEM FOR MECHANICAL ANALYSIS}

In this section we address a new strategy for the mechanical analysis of welding processes with one eye on its industrial applicability. This strategy is based on the Smart-GFEM, presented above, including other techniques of model order reduction such as the proper orthogonal decomposition (POD) and the proper generalized decomposition (PGD). The strategy, schematized in Fig. 4, is composed of two stages, off-line and on-line stages, with different blocks that are elaborated below.

In the off-line stage, for each family of welding processes a POD analysis is carried out. A family of processes is determined by certain technological, material and geometric parameters which are constant for all the snapshots while the others may vary. The aim is to find, for each family, a reduced basis for any thermal history in the local material volume. Thus, for any point at any instant, the temperature can be expressed as $T=\sum_{i}^{N} \alpha_{i} \varphi(\boldsymbol{x}, t)$, where the set of POD modes $\varphi_{i}(\boldsymbol{x}, t)$ constitutes the reduced basis. The number of modes $N$ should be small, let us say less than ten, and the different families of processes should be created to achieve this goal. Optimizing the selection of families of welding processes is the subject of current research.

The material volume must be large enough to capture the strong thermal gradients around the heat source. These thermal snapshots can be computed using the Smart-GFEM. However, we use the commercial code SYSWELD because all the metallurgical models are already included, which are not yet available in our Smart-GFEM implementation.

Then, a set of elasto-plastic problems are solved in the material local volume imposing homogeneous essential boundary conditions. We search the stress field $\sigma_{0}$ generated by each thermal history defined by a point in the $N$-dimensional $\alpha$-space. The sampling in this space can be performed using different strategies [12, 13]. Then, a 


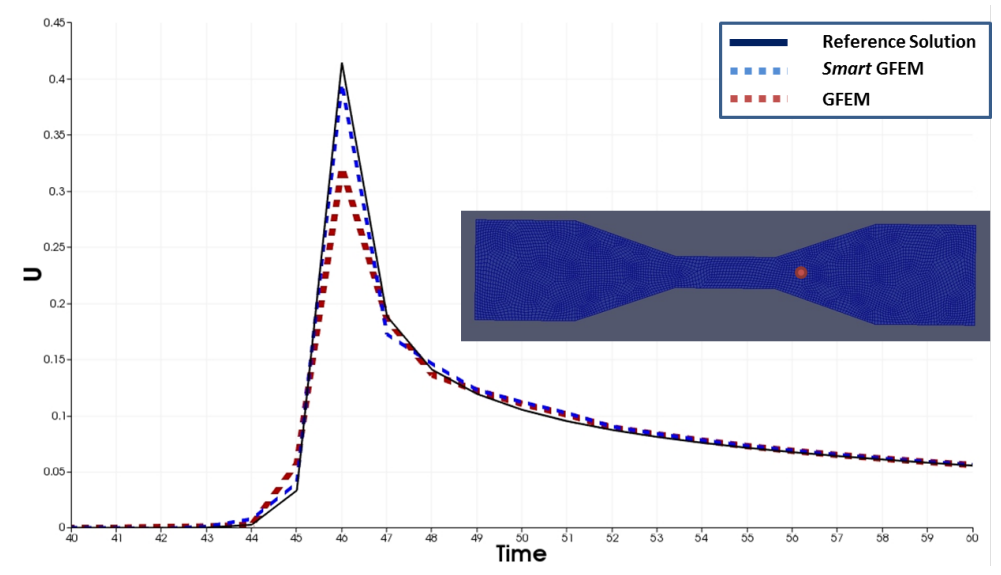

FIGURE 3: Thermal history of a material point

manifold of the resultant stress field, $\sigma_{0}$ is built in the same $\alpha$-space:

$$
\sigma_{0}=\sigma_{0}\left(\alpha_{1}, \alpha_{2}, \cdots, \alpha_{N}\right) .
$$

Finally, a computational vademecum for an elastic problem in the local material volume is constructed. The stress field $\sigma_{0}\left(\alpha_{1}, \alpha_{2}, \cdots, \alpha_{N}\right)$ constitutes the only force (body force), and the set of $\alpha_{i}$ is introduced as extra-coordinates. The boundary conditions are also considered as extra-coordinates. This vademecum provides the local displacement field $\boldsymbol{u}_{l}$, for any $\boldsymbol{\sigma}_{0}$ stress and for any displacement boundary conditions, $\boldsymbol{u}_{\Gamma}$,

$$
\boldsymbol{u}_{l}=\boldsymbol{u}_{l}\left(\sigma_{0}, \boldsymbol{u}_{\Gamma}\right)
$$

In other words, it provides the local mechanical response of the local volume for any essential boundary conditions and for any thermal history introduced in the local volume, when this thermal history is represented in the reduced basis,

$$
\boldsymbol{u}_{l}=\boldsymbol{u}_{l}\left(\alpha_{1}, \alpha_{2}, \cdots, \alpha_{N}, \boldsymbol{u}_{\Gamma}\right)
$$

This local solution $\boldsymbol{u}_{l}$ constitutes the parametric enrichment, also denoted by $\boldsymbol{\phi}$, of our Smart-GFEM formulation. This parametric enrichment is particularized at each time step in the on-line stage,

In the on-line stage, firstly, the thermal problem is solved using SYSWELD. Then, the thermal history of each point located near to the welding line is projected onto the reduced basis composed of the set of $\varphi_{i}$ modes, obtaining the coefficients $\alpha_{i}$. With these coefficients and the values of the displacement field at the border of the enrichment volume $\boldsymbol{u}_{\Gamma}$, a tailored enrichment function is computed. This enrichment function is nothing but the particularization of the vademecum (6) at the current time-step. In practice, the values of $\boldsymbol{u}_{\Gamma}$ used in this particularization are those obtained at the previous time step.

Once the enrichment function is obtained, the traditional global mechanical problem is solved, but in a coarse mesh. This is possible due to the fact that the approximation space in the heat and mechanical affected zone has improved approximation properties as a result of the tailored enrichment added. It should be noticed that, the enrichment region, as defined in the previous section, is a volume attached to the heat source. However, we are interested in the thermal history and metallurgical composition of material points. Moreover, it is not only the nodes close to the heat source at each instant must be enriched. Additionally, all the material nodes that are near to the weldline must be enriched in order to compute the residual stress and induced deformations in the global piece. Thus, the relation between our enrichment volume attached to the source and the material points of the global piece should be clarified. We discuss briefly these technical aspects in the following section.

\section{The importance of the reference frame}

For the mechanical problem, since an elasto-plastic thermal model is solved, it may seem natural to consider a reference frame attached to the material points, i.e., a Lagrangian reference frame. However, due to the fact that we are 


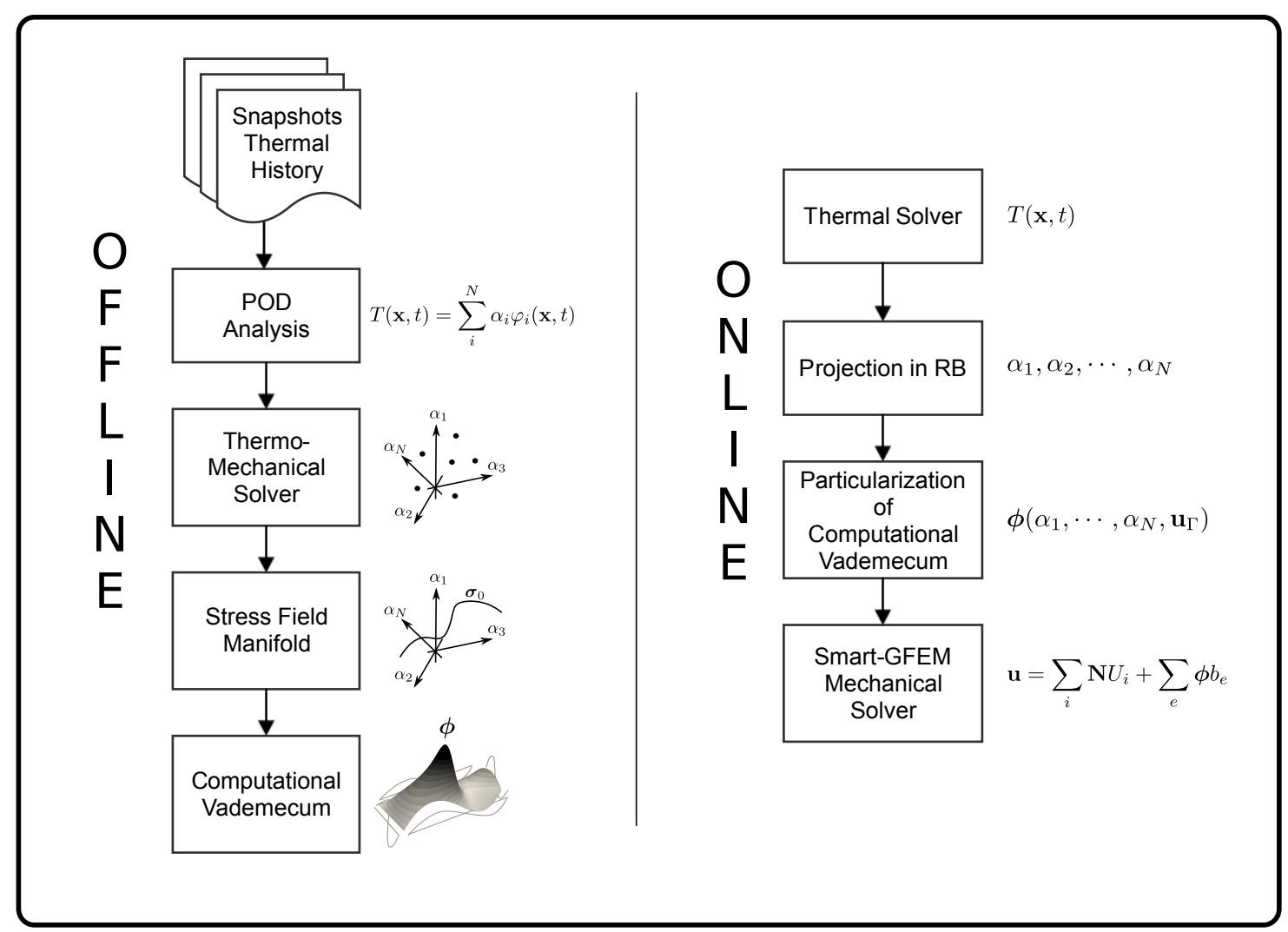

FIGURE 4: Smart-GFEM FOR MECHANICAL ANALYSIS

neglecting the transient effects in our analysis, the Eulerian frame can take advantage of the steady-state conditions that may exist with respect to the moving heat source. Thus, it could be more appropriate from the computational point of view to construct the set of snapshots and to extract the reduced basis in an Eulerian reference frame attached to the moving heat source.

Obviously, the mathematical model of the process should be conveniently modified to include this fact. Let us consider a material point in the Lagrangian reference frame $\boldsymbol{X}$ and our enrichment volume attached to a heat source with an steady velocity $\boldsymbol{v}$. The coordinates of any point $\boldsymbol{x}$ in the reference frame of the moving volume are related to the material points through the following expression:

$$
x=X-v t
$$

Thus, the transformation between any field $\varphi(x)$ computed in the off-line stage in the reference frame of our enrichment volume to the field $\hat{\varphi}(\boldsymbol{X}, t)$ can be performed as follows:

$$
\begin{gathered}
\hat{\varphi}(X, t)=\varphi(X-v t), \\
\nabla_{X} \hat{\varphi}(X, t)=\nabla_{x} \varphi(x), \\
\frac{\partial \hat{\varphi}(X, t)}{\partial t}=-\frac{\partial \varphi(x)}{\partial x} \cdot v,
\end{gathered}
$$

where $-\frac{\partial \varphi(x)}{\partial x} \cdot v$ is the spatial derivative of $\varphi$ along the streamline. The interested reader can find all the details of a thermo-mechanical formulation for welding in this reference frame in [14]. This formulation is already implemented in SYSWELD, and it was used to obtain the set of snapshots for each family of welding processes.

It is worth highlighting the fact that, in the Smart-GFEM, the Eulerian reference frame is only used in the offline process, because we are interested in the local behavior of the solution. Once the computational vademecum 
is constructed, in the on-line stage, the global problem is solved in a Lagrangian reference frame, a much more convenient frame for introducing the global geometry and computing the residual stresses and induced deformations of the entire piece.

\section{CONCLUSIONS AND FUTURE WORK}

In this work we have presented a new strategy to perform efficient thermal and mechanical analyses of welding processes using the Smart-GFEM. We have seen that this technique allows us to precompute off-line complete fundamental solutions to improve the local approximation properties of our generalized finite element method. These vademecums take into account explicitly the parameters of the processes and the conditions around the local region. Even if some of these parameters vary during the process, the enrichment space is updated in the on-line stage with no computational cost.

The construction of the vademecums is more efficient if performed in an Eulerian reference frame. Later, the in-line stage, this field is pass to the material point with the transformation given.

Even when the Smart-GFEM has been validated for the thermal analysis, the validation of the proposed strategy for the mechanical one is still a work in progress.

\section{ACKNOWLEDGMENTS}

The authors wish to thank the ESI Group for its financial support through the ESI group - ECN Chair. In addition, E. Cueto gratefully acknowledges the financial support by the Spanish Ministry of Economy and Competitiveness through Grant number CICYT DPI2014-51844-C2-1-R, and by the Regional Government of Aragon and the European Social Fund.

\section{REFERENCES}

[1] J. Bergheau and R. Fortunier, Finite Element Simulation of Heat Transfer (Wiley, 2013).

[2] F. Chinesta, A. Leygue, B. Bognet, C. Ghnatios, F. Poulhaon, F. Bordeu, A. Barasinski, A. Poitou, S. Chatel, and S. Maison-Le-Poec, International journal of material forming 7, 81-92 (2004).

[3] F. Chinesta, A. Leygue, F. Bordeu, J. V. Aguado, E. Cueto, D. González, I. Alfaro, A. Ammar, and A. Huerta, Archives of Computational Methods in Engineering 20, 31-59 (2013).

[4] F. Chinesta, P. Ladeveze, and E. Cueto, Archives of Computational Methods in Engineering 18, 395-404 (2011).

[5] D. Canales, A. Leygue, F. Chinesta, G. D., E. Cueto, E. Feulvarch, J. Bergheau, and A. Huerta, In press 7, 81-92 (2015).

[6] C. Duarte and D.-J. Kim, Computer Methods in Applied Mechanics and Engineering 197, 487-504 (2008).

[7] J. Melenk and I. Babuška, Computer Methods in Applied Mechanics and Engineering 139, 289-314 (1996).

[8] T. Strouboulis, I. Babuška, and K. Copps, Computer methods in applied mechanics and engineering 181, 43-69 (2000).

[9] J. Melenk, “On generalized finite element methods," Ph.D. thesis, University of Maryland 1995.

[10] R. Merle and J. Dolbow, Computational mechanics 28, 339-350 (2002).

[11] W. Aquino, J. Brigham, C. Earls, and N. Sukumar, International journal for numerical methods in engineering 79, p. 887 (2009).

[12] P. A. Maday, Y. and G. Turinici, Journal of Scientific Computing 17, 437-446 (2002).

[13] R. D. V. K. M. L. M. Y. P. A. Prudhomme, C. and G. Turinici, Journal of Fluids Engineering 124, 70-80 (2001).

[14] J. Shanghvi and P. Michaleris, International journal for numerical methods in engineering 53, 1533-1556 (2002). 